\title{
Automated SEM Analysis in Industrial Process Control and Scientific Research
}

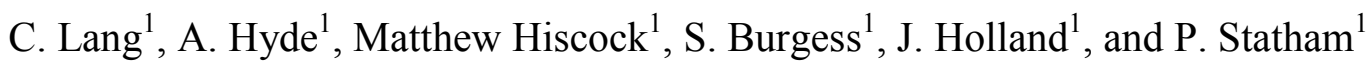 \\ 1. Oxford Instruments NanoAnalysis, Halifax Road, High Wycombe, HP12 3SE, UK.
}

While automated SEM analysis has been used for specific tasks such as gunshot residue analysis and technical cleanliness analysis for many years, recent advances in hardware and software make it a compelling proposition for a much wider group of researchers. Automated analysis in this context refers to solutions that enable users to define analysis tasks that are then carried out repeatedly, sometimes over large sample areas (Figure 1) or even multiple samples. The automation solution comprises control of the microscope stage, electron beam and the analysis equipment such as an EDS detector during a run as well as data reporting tools. Analysis areas are identified automatically based on user defined criteria involving sophisticated image processing. Data from both the EDS detector and imaging detectors containing both morphological and compositional information can then be combined to classify analysis areas according to set criteria.

The benefit of using automated runs over manual analysis are multiple such as

- elimination of user bias in selecting analysis areas and interpreting results

- enhanced accuracy in moving and positioning the microscope stage when covering large sample areas

- solution of 'needle in a haystack' problems by systematically searching samples for rare features

- $\quad$ economical use of SEM time by setting up runs over night or at weekends

- rapid knowledge transfer of sample analysis procedures between different users or across sites by defining standard recipes.

In earlier solutions, the set-up requirements for such an automated run used to be a lengthy process involving trial and error, with the complexity of the setup process sometimes outweighing the benefits of automated analysis over manual analysis. Therefore, automated analysis has so far been confined largely to particle analysis applications in industrial process control and forensics where the time to locate each particle manually would be too arduous. However, new software reduces the set-up times significantly and the use of set-up wizards and recipes renders setting up an automated run a task that can easily be achieved by even novice users.

While solutions dedicated to one particular particle analysis task are offered by a variety of vendors, they are generally not flexible enough to cater for evolving automatic analysis needs. Oxford Instruments has integrated a variety of automated analysis routines in its AZtec Microanalysis Suite. Through the use of setup wizards, complex tasks have been broken down into a logical sequence of steps so that setting up an automated analysis run becomes quick and easy to do.

We show how automated runs can be used to analyzing inclusions in metals (Figure 2a), determine the phase distribution, association (Figure 2b) and porosity of geological samples as well as analyze particulate samples containing wear debris from combustion engines. Furthermore we explore the limits of automated analysis when it comes to nanoscale particles. There we will explore the of use low-kV data to minimize the interaction volume and still identify particles and compositional features reliably 
by using large area X-Max ${ }^{\mathrm{N}}$ SDDs. We also demonstrate how automated analysis can be used to solve a needle in a haystack problem on a sample containing a single gold particle amongst tens of thousands of other particles. While a number of important morphological and compositional parameters are available directly from the data recorded during the automated run, we show how we can relocate the particle and study it in more detail using alternative microanalysis techniques such as WDS to reveal even more information about the sample.

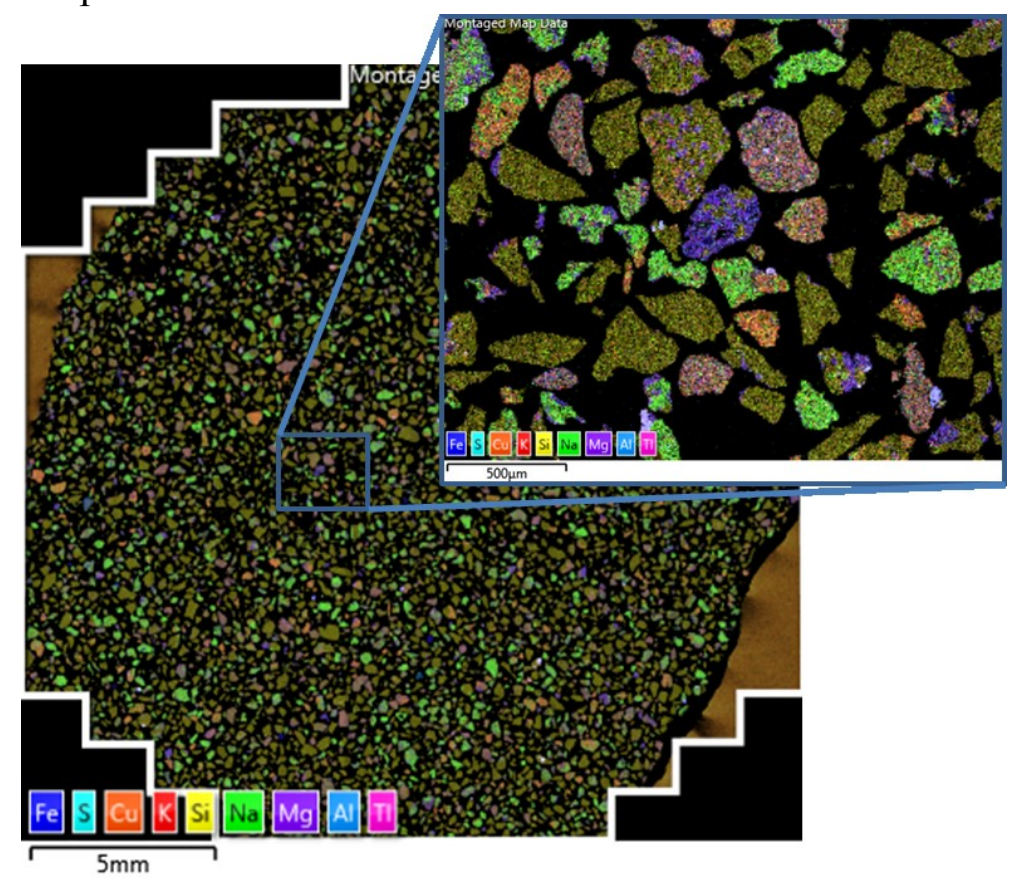

Figure 1. shows a large area map of a typical sample used for particle analysis containing several thousand particles. All data was acquired and processed automatically, providing a wealth of data on an individual particle basis.

(a)

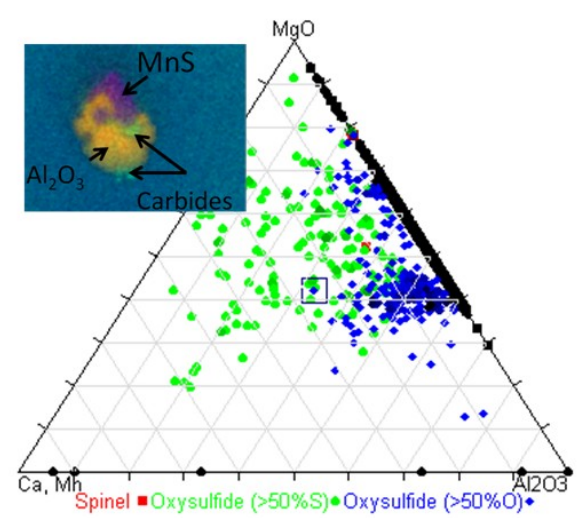

Figure 2. Shows statistical data obtained by automated SEM analysis. (a) shows a plot of different types of steel inclusions which were analyzed and classified automatically. The inset shows an X-Ray map of an individual inclusion. (b) shows the association diagram of sphalerite calculated for a sample containing more than 30000 mineral particles. 